\title{
2 Layer-by-layer bioassembly of cellularized polylactic acid porous membranes for bone tissue engineering
}

\author{
Vera Guduric $\mathbb{B}^{1,2} \cdot$ Carole Metz $^{1} \cdot$ Robin Siadous $^{1} \cdot$ Reine Bareille $^{1} \cdot$ \\ Riccardo Levato $^{3,4} \cdot$ Elisabeth Engel $^{3} \cdot$ Jean-Christophe Fricain $^{1} \cdot$ Raphaël Devillard $^{1}$. \\ Ognjan Luzanin ${ }^{2} \cdot$ Sylvain Catros ${ }^{1}$
}

Received: 6 November 2016 / Accepted: 15 March 2017

(C) Springer Science+Business Media New York 2017

Abstract The conventional tissue engineering is based on seeding of macroporous scaffold on its surface ("top-down" approach). The main limitation is poor cell viability in the middle of the scaffold due to poor diffusion of oxygen and nutrients and insufficient vascularization. Layer-by-Layer (LBL) bioassembly is based on "bottom-up" approach, which considers assembly of small cellularized blocks. The aim of this work was to evaluate proliferation and differentiation of human bone marrow stromal cells (HBMSCs) and endothelial progenitor cells (EPCs) in two and three dimensions (2D, 3D) using a LBL assembly of polylactic acid (PLA) scaffolds fabricated by $3 \mathrm{D}$ printing. 2D experiments have shown maintain of cell viability on PLA, especially when a co-cuture system was used, as well as adequate morphology of seeded cells. Early osteoblastic and endothelial differentiations were observed and cell proliferation was increased after 7 days of culture. In $3 \mathrm{D}$, cell migration was observed between layers of LBL constructs, as well as an osteoblastic differentiation. These results indicate that LBL assembly of PLA layers could be suitable for BTE, in order to promote homogenous cell distribution

Vera Guduric

vera.guduric@inserm.fr

1 Biotis, Inserm U1026, Université Bordeaux Segalen, 146 rue LéoSaignat, Case 45, Bordeaux Cedex 33076, France

2 Fakultet Tehnickih Nauka, Univerzitet u Novom Sadu, Trg Dositeja Obradovica 3, Novi Sad 21000, Serbia

3 Biomaterials for Regenerative Therapies Group, Institute for Bioengineering of Catalonia (IBEC), Barcelona, Spain

4 Department of Orthopedics, University Medical Center Utrecht, Utrecht, The Netherlands inside the scaffold and gene expression specific to the cells implanted in the case of co-culture system.

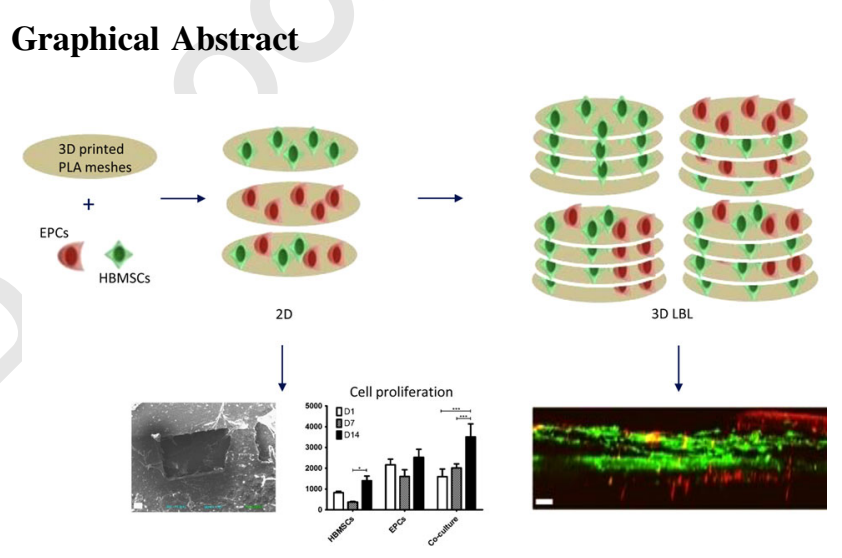

\section{Introduction}

A typical bone tissue engineering (BTE) approach requires cells specific to the bone tissue, biochemical growth factors as well as porous biocompatible scaffold [1]. The role of the scaffold is to provide a support for cell proliferation and differentiation and it must possess specific features regarding pore diameters, porosity and microscopic dimensions, as well as adequate osteoconductive and osteoinductive properties [2]. There are different biomaterials being used for BTE nowadays, such as calcium phosphates, metals, hydrogels, polymers or their combination [3-9]. Different groups have recently used scaffolds made of polylactic acid (PLA) as a support for bone regeneration. Pure PLA scaffolds can be used [10, 11] while coated PLA [12] and PLAbased composite materials have also been described [9, 1316]. The FDA has approved PLA for different biomedical 
applications, and it has proven adequate osteoconductive and osteoinductive properties for bone applications. Different types of human and animal cells have shown high ability to attach onto PLA scaffolds [17-19]. This polymer has been used to fabricate BTE scaffolds using several rapid prototyping (RP) methods, mostly by fused deposition modeling (FDM) [12], and 3D printing [20-22].

Conventional TE approach is based on the seeding of macroporous scaffold on its surface ("Top-Down" = TD), resulting in many cases in poor cell viability inside the scaffold, because it's difficult for cells and nutrients to penetrate and survive in the core of the scaffold [23]. "Bioassembly" is based on self-induced assembly of cellularized building blocks and might also be called a "Bottom-Up" (BU) approach [24]. The main advantage of this approach is the possibility to seed different cell types onto one scaffold, which may lead to a homogeneous cell colonization and proliferation inside the scaffold. Layer-bylayer (LBL) assemblies of cellularized porous biomaterials may be used to fabricate cellularized constructs for bone tissue regeneration. The choice of the right order of layers plays an important role in order to obtain the best final implantable construct [25]. It was shown before that the combination of human bone marrow stromal cells (HBMSCs) and human umbilical vein endothelial cells (HUVECs) in alternating layers of cell sheets enables a high vascularization subctunaeously in mice [26]. Moreover, angiogenic factors secretion was augmented when alternates layers of mesenchymal stem cells and endothelial cells are stacked [27]. It was shown previously that it is possible to control the microenvironment inside the scaffold when using LBL approach since it enables the control of each layer accurately [28]. Another experiment based on LBL paper-stacking using adipose derived stem cells (ADSCs) and PCL/gelatin in vivo has shown that the LBL approach gave a promising osteogenic-related gene expressions [29]. We have already tested this method with MG63 cells transduced with Luciferase gene and PCL electrospun scaffold biopapers. Luciferase tracking with photon-imager displayed that cell proliferation was increased when the materials and cells were stacked layer-by-layer [30].

Concerning the cellular component of bone tissue engineering, it is already known that endothelial progenitor cells (EPCs) can modulate differentiation properties of mesenchymal stem cells (MSCs) in a coculture system [31]. PLA has already been used as a scaffold for MSCs and EPCs isolated from the rat [32] but there are no data available for the coculture of human endothelial and osteoblastic cells on this material. The use of PLA scaffold membranes to support cell culture could improve the manipulation and mechanical properties of such constructs.

The aim of this work was to build PLA membranes cellularized with human osteoprogenitors and endothelial progenitor cells and to evaluate its properties in vitro in 2and 3-dimensions

\section{Materials and methods}

\subsection{Preparation of PLA membranes}

PLA membranes were fabricated at the Institute for Bioengineering of Catalonia (IBEC) by direct 3D printing method, an additive RP method based on the extrusion of PLA dissolved in chloroform through a nozzle. We have used a 3Dn-300, Sciperio/nScrypt (Inc. Orlando, Florida) printer for this study. The PLA solution was prepared by dissolving a Poly(95 L/5DL) lactic acid (Corbion Purac) in chloroform $(5 \% \mathrm{w} / \mathrm{v})$ at $45{ }^{\circ} \mathrm{C}$ during $24 \mathrm{~h}$ and then syringes of $5 \mathrm{~mL}$ were filled, closed with paraffin film and stored at $-20{ }^{\circ} \mathrm{C}$ before use. The printing process was controlled using a tuned motor speed and pressure, in order to be adapted to viscosity of the solution. The motor speed was 3 $\mathrm{mm} / \mathrm{s}$ and the pressure was between 40 and 80 psi. G27 nozzles were used for extrusion. In order to be used for experiments, raw membranes $\left(4 \mathrm{~cm}^{2}\right)$ were cut with a tissue punch into $8 \mathrm{~mm}$ diameter circles.

Before cell culture experiments, PLA membranes were rinsed with phosphate buffered saline (PBS) $0.1<\mathrm{pH} 7.4$ (Gibco) and sterilized in a solution of ethanol $70 \%(\mathrm{v} / \mathrm{v})$ during $30 \mathrm{~min}$. Then, the membranes were rinsed twice with PBS. A small amount of 2\% agarose (A9539-250G SigmaAldrich, St Louis, MO, USA) prepared in PBS was placed in each well before placing the membranes in order to prevent cell adhesion on tissue culture plastic (TCP). The membranes were rinsed with culture media during $24 \mathrm{~h}$ before seeding the membranes with cells. All experiments were performed in 48-well plates (Corning Inc-Life Sciences, Durham, NC, USA).

\subsection{Cell isolation and tagging}

Two types of human primary cells were used in this study: human bone marrow stromal cells (HBMSCs) were isolated from bone marrow retrieved during surgical procedures (Experimental Agreement with CHU de Bordeaux, Etablissement Français du Sang, agreement CPIS 14.14). Cells were separated into a single suspension by sequential passages through syringes fitted with 16-, 18- or 21-gauge needles. After the centrifugation of $15 \mathrm{~min}$ at $800 \times \mathrm{g}$ without break at room temperature, the pellet was resuspended with $\alpha$-Essential Medium ( $\alpha$-MEM; Invitrogen) supplemented with $10 \%(\mathrm{v} / \mathrm{v})$ fetal bovine serum (FBS) [33]. Endothelial Progenitor Cells (EPCs) were isolated from $30 \mu \mathrm{L}$ of diluted cord blood (Experimental Agreement with CHU de Bordeaux, Etablissement Français du Sang, agreement CPIS 
14.14) in $1 \mathrm{X}$ PBS and $2 \mathrm{mM}$ ethylene diaminetetraacetic acid (EDTA, Sigma-Aldrich, St Louis, MO, USA). $15 \mathrm{mM}$ of Histopaque solution (Sigma-Aldrich) was added. Then centrifugation was performed at $400 \mathrm{~g}$ for $30 \mathrm{~min}$ and the ring of nuclear cells was removed and washed several times with $1 \times$ PBS and $2 \mathrm{nM}$ EDTA. At the end, cells were cultured in endothelial cell growth medium-2 (EGM-2, LonzaVerviers, France) with supplements from the kit and 5\% (v/ v) FCS (GIBCO Life Technologies, Karlsruhe, Germany) on a 12-well cell plate. The cell plate was coated with collagen type I (Rat Tail, BD Biosciences). Non adherent cells were removed at Day 1 and media was changed every other day [34]. The medium for endothelial cells growth contained 5\% FBS, $0.1 \%$ human epidermal growth factor (hEGF), $0.04 \%$ Hydrocortison, 4\% human fibroblastic growth factor-b (hFGF-b), $0.1 \%$ vascular endothelial growth factor (VEGF), 0.1\% R3 insulin-like growth factor-1 (R3-IGF-1) $0.1 \%$ ascorbic acid, $0.1 \%$ gentamicin, amphotericin B (GA) (Lonza-Verviers, France). Both, HBMSCs and EPCs were incubated in a humidified atmosphere of $95 \%$ air, $5 \% \mathrm{CO}_{2}$ at $37^{\circ} \mathrm{C}$. The culture medium was changed every other day.

To evaluate the cell migration during LBL 3D experiments, both types of cells were tagged with fluorescent proteins. HBMSCs were tagged with green fluorescent protein (GFP) which exhibits a green fluorescence when exposed to light in the blue or ultraviolet range. EPCs were tagged with Td-Tomato, which exhibits a red fluorescence when exposed to the light in green range [35]. The lentiviral vectors contained GFP or Td-Tomato protein gene under the control of the MND (for GFP) or phosphoglycerate kinase (PGK) promoter (for Td-Tomato) for cell labeling. $2 \times 10^{5}$ freshly trypsinized HBMSCs ou EPCs (low subculturing) in suspension were mixed with $6 \times 10^{6}$ viral particles (MOI for GFP: 15; MOI for Td-Tomato: 30) for viral transduction (multiplicity of infection). After $24 \mathrm{~h}$ in culture, virus-containing medium was replaced by a fresh one to provide the cell growth. Medium was changed every other day.

\subsection{Cell seeding and characterization in 2D}

\subsubsection{Cell seeding in $2 D$}

PLA membranes were stabilized on the agarose with glass rings in order to avoid the floating of membranes in the culture media. HBMSCs and EPCs were seeded onto membranes as mono- (HBMSCs 50,000 cells $/ \mathrm{cm}^{2}$, EPCs 100,000 cells $/ \mathrm{cm}^{2}$ ) and co-cultures (HBMSCs $25,000 / \mathrm{cm}^{2}$ + EPCs 50,000 cells $/ \mathrm{cm}^{2}$ ). Culture media were changer every other day.

All 2D experiments were performed on PLA membranes seeded with different combinations of human primary cells
(1 seeded membrane $=1$ sample). Examined time points were Day 1, Day 3, Day 7, Day 14 and Day 21.

\subsubsection{Cell characterization in $2 D$}

2.3.1.1 Live-dead assay The viability of the cells seeded on PLA membranes was tested by Live-Dead assay (LD, Life Technologies), which was based on acetoxymethylester of calcein (Calcein-AM) and ethidium homodimer-1 (EthD-1) [36-38]. Calcein-AM was cleaved in the cytoplasm by esterase and thus indicated live cells showing the green fluorescence. EthD-1 enters cells with damaged membranes and binds to nucleic acids, producing a red fluorescence of dead cells. The assay was performed by removing the culture media, rinsing the seeded PLA membrane with Hanks' balanced salt solution (HBSS, GIBCO) and addition of the solution of Calcein-AM and EthD-1 diluted in Hanks'. The solution was incubated during $15 \mathrm{~min}$ in a humidified atmosphere of $95 \%$ air, $5 \%$ $\mathrm{CO}_{2}$ at $37^{\circ} \mathrm{C}$. Fluorescence was observed with confocal scanning microscopy (Leica, TSC SPE DMI 4000B) with LAS-AF (Leica Advanced Suite-Advanced Fluorescence) software.

2.3.2.2 Quantification of the area covered by cells LiveDead images obtained by confocal microscope were used to calculate areas covered by live or dead cells by ImageJ (https://imagej.nih.gov/ij/).

For each condition (mono- or co-cultures) and for each time point, we have selected five images (four close to the borders at the ends of perpendicular axes and one in the middle) to quantify the cell area covered by cells. This lead to a total of 45 images quantified. Color channels (green and red) were split for each image and percentage of covered areas were calculated for each color. Statistical analyses were performed with GraphPad Prism 6 software using a two way ANOVA and Bonferroni tests.

2.3.3.3 Scanning electron microscopy Cell morphology was observed with a microscope Hitachi, S-2500 scanning electron microscope (SEM). After 14 days of cell culture onto PLA membranes, the samples were fixed with paraformaldehyde (PFA) 4\% and dehydrated in graded ethanol (EtOH) solution $(30,50,70,90,100 \%)$ and then in dexamethylsilazan and air dried, followed by gold coating. The accelerating voltage used for the observation was $12 \mathrm{kV}$ and the samples were observed with magnification $\times 80$ and $\times 200$. Pictures were acquired using MaxView ${ }^{\circledR}$ and SamX® softwares.

2.3.4.4 CyQuant assay Cell proliferation on PLA was evaluated with CyQuant ${ }^{\circledR}$ Cell Assay kit (In vitrogen C7026). This assay was based on fluorescent quantification
203

204

205

206

207

208

209

210 
of one protein which binded to cell DNA. The culture media was removed at each time point and culture plates were frozen and kept at $-80^{\circ} \mathrm{C}$ to process all samples together. Finally, all plates were left at the room temperature for thawing. The lysis solution was first added in all samples and then $200 \mu \mathrm{l}$ of the buffer were added following the manufacturer's instructions. All samples were transferred in 96-well plates and mixed for 2-5 min in dark. The fluorescence of the solutions was measured at 480 and $520 \mathrm{~nm}$ using Victor X3 2030 Perkin Elmer.

2.3.5.5 Immunofluorescent analysis The EPCs monocultures and the co-cultures HBMSCs + EPCs on PLA membranes were fixed with $4 \%$ (w/v) Paraformaldexyd (PFA) at $4{ }^{\circ} \mathrm{C}$ during $15 \mathrm{~min}$ and permeabilized with Triton $\mathrm{X}-1000.1 \%(\mathrm{v} / \mathrm{v})$ during $10 \mathrm{~min}$. Endothelial phenotype was observed using intracellular marker von Willebrand Factor (vWF). The samples were incubated $1 \mathrm{~h}$ in PBS containing $1 \%(\mathrm{w} / \mathrm{v})$ Bovine serum albumin (BSA, Eurobio, France) before incubation with primary antibody. VWF primary antibody (Rabbit) was diluted in PBS $1 \times$ with $0.5 \%$ (w/v) BSA at 1/300 (Dako, Glostrup, Denmark). The primary antibody was incubated $1.5 \mathrm{~h}$ at the room temperature. Then, the cells were rinsed with PBS and incubated with the secondary antibody: Alexa 488-conjugated goat anti-rabbit IgG diluted at 1/300. Subsequently, cells were washed with PBS and incubated with the nuclear probe DAPI (4', 6'-diamino-2-phenylindole, FluoProbes $5 \mathrm{mg}$ $\mathrm{ml}^{-1}$, dilution 1:5000) for $10 \mathrm{~min}$ at room temperature, in order to label the nucleus in blue. The lasers used were 488 nm (green), $561 \mathrm{~nm}$ (red) and $405 \mathrm{~nm}$ (blue). The observations were performed at $100 \times$ magnification and the pictures were taken every $2.4 \mu \mathrm{m}$ in " $\mathrm{z}$ " orientation. The 3D reconstruction was performed with LAS-AF (Leica Advanced Suite-Advanced Fluorescence) software.

2.3.6.6 Alkaline phosphatase (ALP) assay Intracellular ALP activity was detected as an early osteoblastic marker. We have used the Ackerman technique, which is based on conversion of a colorless p-nitrophenyl phosphate to a colored $\mathrm{p}$ nitrophenol (Sigma diagnostic kit, Aldrich). Three different conditions were tested: (1) mono-culture (HBMSCs) with induction media $(\alpha$-MEM $+1 / 1000$ dexamethasone, $1 / 10,000$ ascorbic acid, 1/100 $\beta$-glycerolphosphate, Iscove's Modified Dulbecco (IMDM, GIBCO), 10\% SVF); (2) mono-culture (HBMSCs) without induction media ( $\alpha$-MEM alone) and (3) co-cultures ( $\alpha$-MEM + EGM-2 50/50). The samples were fixed with $4 \%(\mathrm{v} / \mathrm{w})$ PFA during $10 \mathrm{~min}$ at $4{ }^{\circ} \mathrm{C}$. Then the samples were stained with alkaline dye (Fast bluse RR salt supplemented with Naphtol AS-MX phosphate alkaline solution $0.25 \%$, Sigma Aldrich) away from light during 30 min. The observations were performed with an optical microscope (Leica DMi 3000 B) connected with a digital camera (Leica DFC $425^{\circ} \mathrm{C}$ ).

\subsection{Layer-by-Layer assembly of cellularized membranes in $3 \mathrm{D}$}

\subsubsection{Layer-by-layer assembly and seeding strategies}

After seeding the PLA membranes in 2D using HBMSCs or EPCs or cocultures of HBMSCs and EPCs, the membranes were stacked Layer-by-Layer (LBL) to obtain a 3D composite material (Fig. 1).

These 3D constructs were prepared by assembling four PLA membranes seeded with human primary cells (HBMSCs alone or coculture of HBMSCs and EPCs) after 3 days of culture in 2D. We have prepared four different types of 3D constructs: Sample "A" consisted of four membranes seeded with HBMSC, samples "B" was made of alternating layers of monocultures of HBMSCs and EPCs, samples "C" were constructed with co-culture membranes and samples " $\mathrm{D}$ " had alternating layers of mono-cultures of HBMSCs and co-cultures (Fig. 1). LBL constructs were first characterized by observing the migration of tagged endothelial cells inside the LBL constructs using two photons microscopy, then the osteoblastic differentiation of the LBL 3D constructs was evaluated using quantitative polymerase chain reaction (qPCR).

\subsubsection{Quantitative real-time polymerase chain reaction (QPCR)}

Osteoblastic differentiation was examined on three different types of LBL constructs: HBMSCs in all four layers of 3D constructs, HBMSCs/EPCs/HBMSCs/EPCs and cocultures
Fig. 1 LBL bio-assembly of PLA membranes seeded with human cells. a HBMSCs/ HBMSCs/HBMSCs/HBMSCs; b HBMSCs/EPCs/HBMSCs/ EPCs; c Cocultures/Cocultures/ Cocultures/Cocultures; d HBMSCs/Coculture/HBMSCs/ Coculture

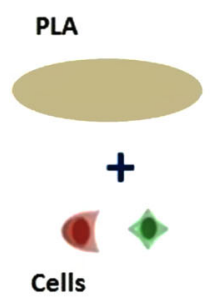

Seeded membranes

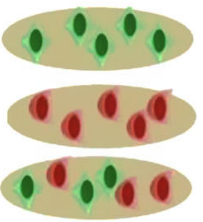
Incubation 3 days
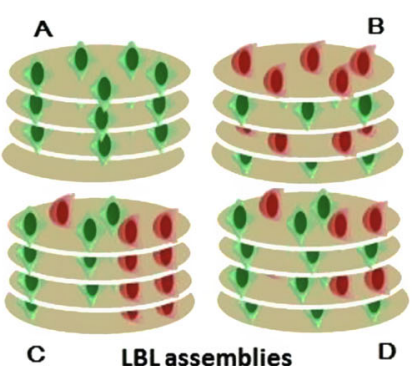
in all four layers (Fig. 1a-c). Total RNA was extracted using the RNeasy Total RNA kit (Qiagen, AMBION, Inc. Austin, Texas, USA), as indicated by the manufacturer and $1 \mu \mathrm{l}$ was used as the template for single-strand cDNA synthesis, using the Superscript pre-amplification system (Gibco) in a $20 \mathrm{ml}$ final volume, containing $20 \mathrm{mM}$ Tris$\mathrm{HCl}, \mathrm{pH} 8.4,50 \mathrm{mM} \mathrm{KCl}, 2.5 \mathrm{mM} \mathrm{MgCl} 2,0.1 \mathrm{mg} / \mathrm{ml} \mathrm{BSA}$, $10 \mathrm{mM}$ DTT, $0.5 \mathrm{mM}$ of each dATP, dCTP, dGTP and dTTP, $0.5 \mathrm{mg}$ oligo(dT) $12-18$ and $200 \mathrm{U}$ reverse transcriptase. After incubation at $42{ }^{\circ} \mathrm{C}$ for $50 \mathrm{~min}$, the reaction was stopped at $70^{\circ} \mathrm{C}$ for $15 \mathrm{~min}$. cDNA $(5 \mu \mathrm{l})$ diluted at a 1:80 ratio was loaded onto a 96-well plate. Real-time PCR amplification was performed using the SYBR-Green Supermix (2' iQ $50 \mathrm{mM} \mathrm{KCl,} 20 \mathrm{mM}$ Tris- $\mathrm{HCl}, \mathrm{pH} 8.4$, $0.2 \mathrm{mM}$ each dNTP, $25 \mathrm{U} / \mathrm{ml}$ iTaq DNA polymerase, $3 \mathrm{mM}$ $\mathrm{MgCl} 2$, SYBR Green I and $10 \mathrm{nM}$ fluorescein, stabilized in sterile distilled water). Primers of investigated genes (Table 1) were used at a final concentration of $200 \mathrm{nM}$. Data were analysed using iCycler IQ software and compared by the $\Delta \Delta \mathrm{CT}$ method. Q-PCR was performed in triplicate for PCR yield validation. Results of relative gene expressions for LBL $B$ and LBL $C$ on the 7th day of culture were expressed to relative gene expression levels of LBL A. Each Q-PCR was performed in triplicate. Data were normalized to P0 (ribosomal protein) mRNA expression for each condition and was quantified relative to Runx2, ALP, OCN and type I collagen (Col1) gene expression. Statistical analysis was performed by Mann Witney test in order to compare the expressions of different gens for $B$ and $C$ LBL constructs.

\subsubsection{Photons microscopy (2PM)}

2PM was used to obtain a large field of view of the samples in $3 \mathrm{D}(450 \mu \mathrm{m})$. We prepared $3 \mathrm{D}$ constructs with HBMSCs tagged with GFP and EPCs tagged with TdT in order to observe the colonization of cells inside the LBL constructs (Fig. 1d). The confocal microscope was a Leica DM6000 TSC SP5 MP. L5 filter was used for green and N3 filter for red fluorescence. HCXIRAPO objective with immersion was used to observe the samples. Argon laser for HBMSCs GFP and DPSS 561 for EPCs TdT. Excitation for HBMSCs GFP was performed at $488 \mathrm{~nm}$ and for EPCs TdT at $561 \mathrm{~nm}$ wavelength.

\section{Results}

\subsection{Cell culture onto a PLA substrate membrane}

\subsubsection{Scaffolds membranes features and cell morphology}

The PLA membranes were $100 \mu \mathrm{m}$ thick and pores diameter was $200 \mu \mathrm{m}$. SEM observations showed the external structure of PLA membranes and struts organization, which revealed that pore size was ranged between 165 and $375 \mu \mathrm{m}$ (Fig. 2a). Considering the PLA membranes loaded with cells, we have observed different cell morphologies of the mono- and co-cultures (Fig. 2b): HBMSCs showed elongated and highly-branched morphology. EPCs were small, rounded cells with filopodia towards PLA membranes. Cells in co-cultures were elongated and branched and covered the membrane pores.

\subsubsection{Cell viability}

Live-Dead experiments were performed in 2D cell culture onto PLA membranes (Fig. 3a). In general, we have observed a large amount of living cells after 14 days of culture. Most of the cells were alive at day 1, with the highest survival rates in mono-cultures of HBMSCs. Few EPCs were present on PLA membranes at Day 1. Coculture samples showed similar cell viability as mono-cultures of HBMSCs at day 1. After 7 days of culture, we observed higher density of live cells in HBMSCs mono-culture samples, which was maintained until day 14. Regarding mono-cultures of EPCs, we did not observe any significant difference in qualitative observations of live and dead cells
Table 1 Primers of investigated genes

\begin{tabular}{ll}
\hline Genes & Primers \\
\hline Ubiquitary ribosomic protein P0 & Forward 5'-ATG CCC AGG GAA GAC AGG GC-3' \\
& Reverse 5'-CCA TCA GCA CCA CAG CCT TC-3' \\
ALP & Forward 5'-AGC CCT TCA CTG CCA TCC TGT-3' \\
& Reverse 5'-ATT CTC TCG TTC ACC GCC CAC-3' \\
COL1A1 & Forward 5'-TGG ATG AGG AGA CTG GCA ACC-3' \\
& Reverse 5'-TCA GCA CCA CCG ATG TCC AAA-3' \\
Runx2 & Forward 5'-TCA CCT TGA CCA TAA CCG TCT-3' \\
& Reverse 5'-CGG GAC ACC TAC TCT CAT ACT-3' \\
OCN & Forward 5'-ACC ACA TCG GCT TTC AGG AGG-3' \\
& Reverse 5'-GGG CAA GGG CAA GGG GAA GAG-3' \\
\hline
\end{tabular}


A
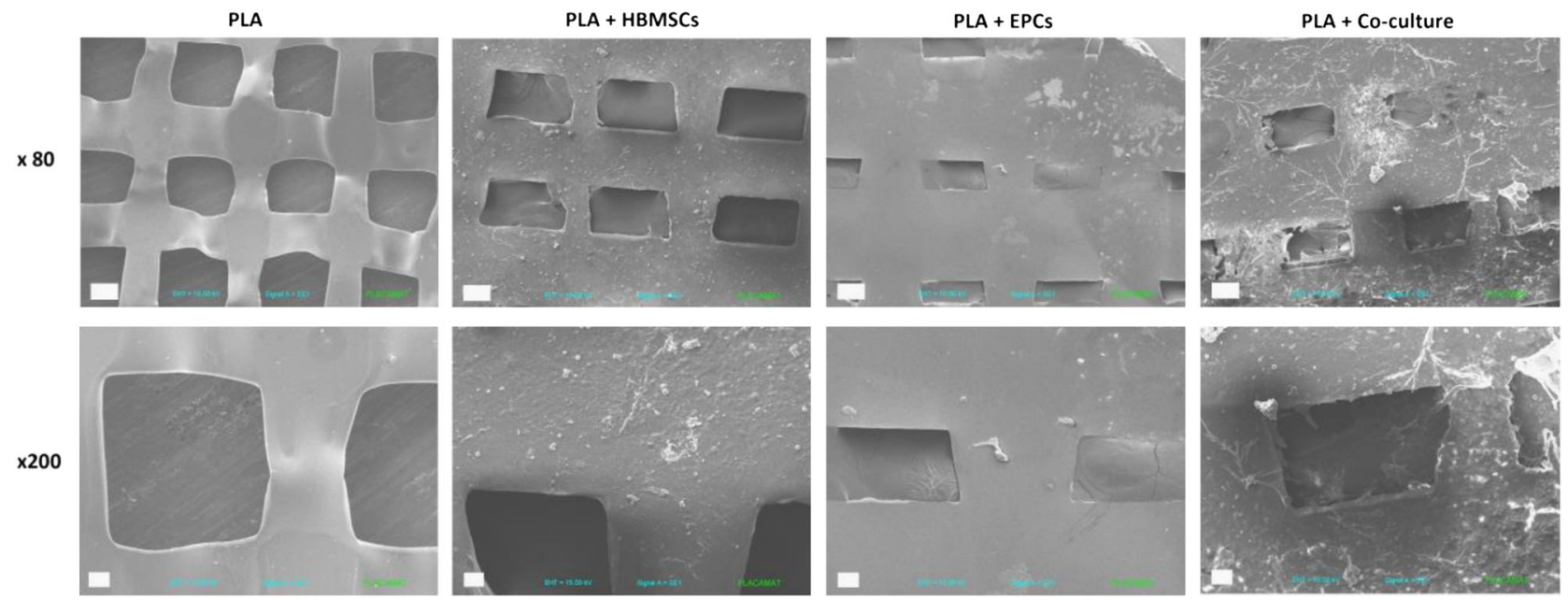

B

Fig. 2 Scanning electron microscopy at Day 14: PLA: control PLA membranes without cells; PLA + HBMSCs: human bone marrow stromal cells cultured on PLA membranes; PLA + EPCs: endothelial progenitor cells cultured on PLA membranes; PLA + Co-cultures: cocultures of HBMSCs and EPCs on PLA membranes. Scale bar is 100 $\mu \mathrm{m}$ for $\times 80$ images and $30 \mu \mathrm{m}$ for $\times 200$ images
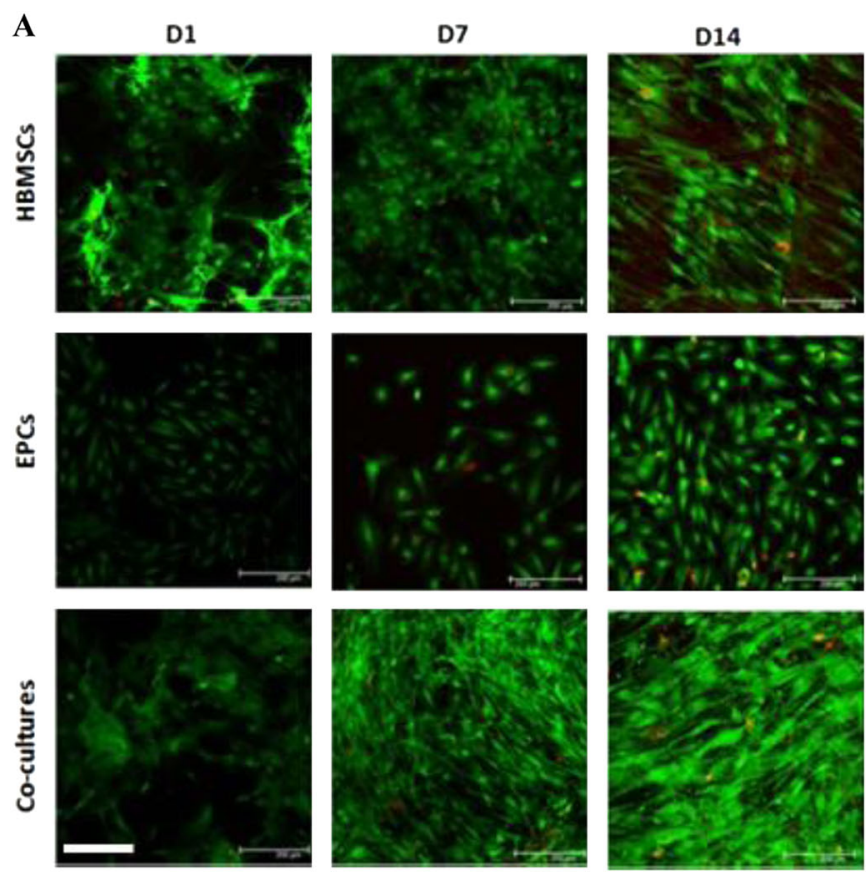

Fig. 3 a Qualitative images of the L/D assay at Day 1, 7 and 14. Scale bar is $200 \mu \mathrm{m}$ for all images; b Statistical results of the \% of total area covered by live cells calculated from five different spots of one

after 7 days, but their population was denser at day 14 . Coculture samples showed a large amount of live cells after 7 days, which was maintained until the day 14. After 14 days, the co-cultures (HBMSCs + EPCs) have shown the highest cell survival.
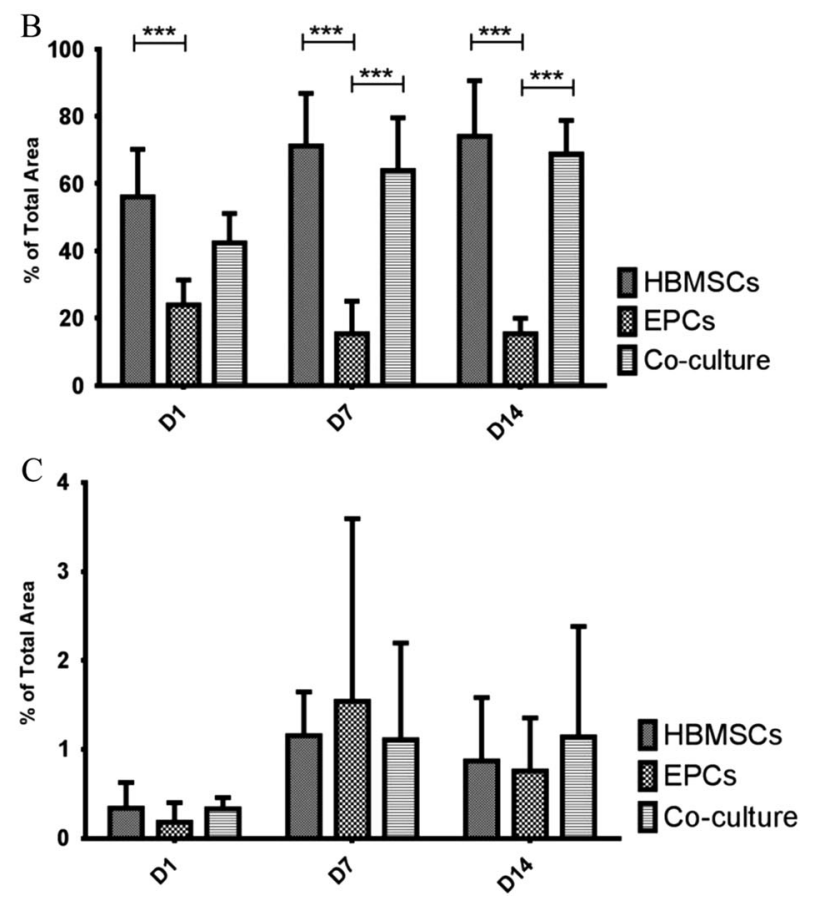

scaffold. $* * * p<0.001$; c Statistical results of the $\%$ of total area covered by dead cells calculated from five different spots of one scaffold

\subsubsection{Quantification of the area covered by cells}

The pictures obtained with confocal microscope after LiveDead assay have been used to quantify the areas covered by live or dead cells, using Image ${ }^{\circledR}$ software. Since the 
Fig. 4 Cell proliferation during 14 days of culture on PLA membranes: mono- and cocultures on PLA. Control experiments were done on tissue culture plastic (TCP). ${ }^{*} p<0.05$, $* * p<0.001, * * * p<0.0001$

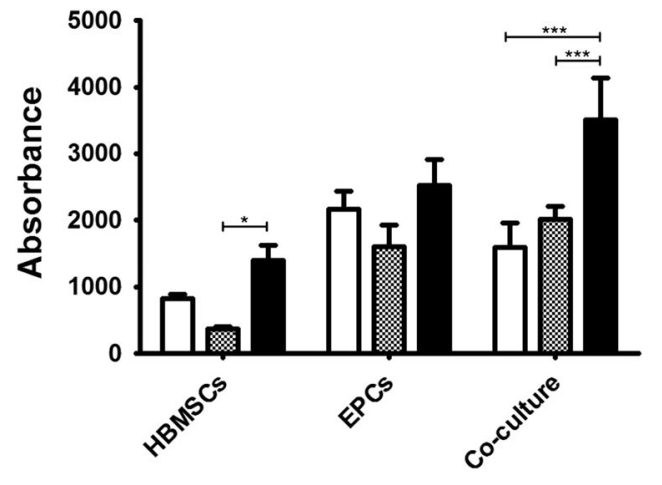

PLA

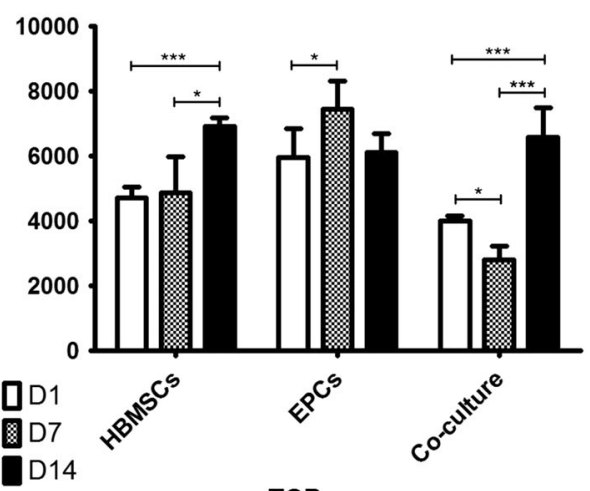

TCP
Calcein-AM colors the cytoplasm of live cells and the EthD-1 colors the nucleus of dead cells, we could not compare the surfaces covered by live to the surfaces covered by dead cells, so we have compared live or dead cells in function of different cell culture conditions. Percentages of total areas of live and dead cells are shown in Fig. 3b and $\mathrm{c}$ respectively. At day 1 , most of the surface covered by live cells was observed in HBMSCs mono-culture samples and it increased with time. The surface of live cells in co-culture systems increased with time as well. Mono-cultures of EPCs did not show an important increase in the surface covered by live cells. There was significantly less EPCs live surface in all conditions compared to HBMSCs and cocultures. Regarding dead cells quantification, no significant difference was observed between all conditions. The highest surface covered by dead cells was observed in EPCs monoculture samples after 7 days.

\subsubsection{Cell proliferation (CyQuant)}

In test samples, cell proliferation assays in two dimensions displayed a global increase of DNA synthesis in all samples with time (Fig. 4). There was no significant difference in the proliferation of EPCs in mono-culture samples during time. DNA synthesis was significantly increased between 7 and 14 days of culture for HBMSCs on the PLA. After 14 days of culture, a significant difference was observed in cell proliferation of co-cultures. Control results (TCP) confirm the significant increase in cell proliferation for all samples after 14 days of culture.

\subsubsection{Cell differentiation}

Endothelial phenotype was characterized by the intracellular marker Von Willebrand Factor (vWF) [39]. DAPI was used to label the nucleus in blue [40]. The vWF (green) and the DAPI (blue) staining were maintained in mono- and co- cultures on PLA during 14 days. Mono-cultures of EPCs on PLA showed a different organization than co-cultures on PLA membranes (Fig. 5a).

Osteoblastic phenotype was evaluated using alkaline phosphatase (ALP) staining. ALP expression was positive in both, mono- and co-cultures (Fig. 5b).

\subsection{Use of cellularized PLA membranes for LBL bio- assembly}

In aim to obtain preliminary results for LBL Bio-Assembly we have characterized the osteoblastic phenotype in $3 \mathrm{D}$ constructs as well as the cell repartition in 3D.

\subsubsection{Phenotype characterization in $3 D$ constructs}

The relative osteoblastic gene expressions at the 7th day of culture of two types of LBL constructs, with different positions of HBMSCs and EPCs in layers., The experiment was performed with LBL constructs with alternating layers of mono-cultures of HBMSCs and EPCs and LBL constructs with co-culture layers. Phenotype characterization was tested for relative gene expression of ALP, RunX2, OCN and Coll as osteoblastic markers (Fig. 6a). LBL construct made of mono-cultures of HBMSCs were used as a control group.

3.2.1.1 Observation of 3D LBL composite materials by 2photons microscopy This experiment was performed in aim to observe the repartition of cells (EPCs) in 3D in LBL constructs. LBL composite materials were prepared to be observed after 14 days of culture using two photons confocal microscopy (2P). The tested sample had alternating layers of monoculture of HBMSCs-GFP and co-cultures (HBMSCs-GFP + EPCs-TdT). We could observe all four layers of 3D constructs and endothelial cells (red fluorescence) were present in all layers (Fig. 6b). 
Fig. 5 Cell differentiation in 2D mono and co-cultures on PLA membranes. The scale is $100 \mu \mathrm{m}$ and it is the same for all images: a endothelial differentiation (vWF in green and DAPI in blue) at Day 14.; b osteoblastic differentiation on Day 14. (PLA poly-lactic acid membranes; $T C P$ tissue culture plastic) (color figure online)
A

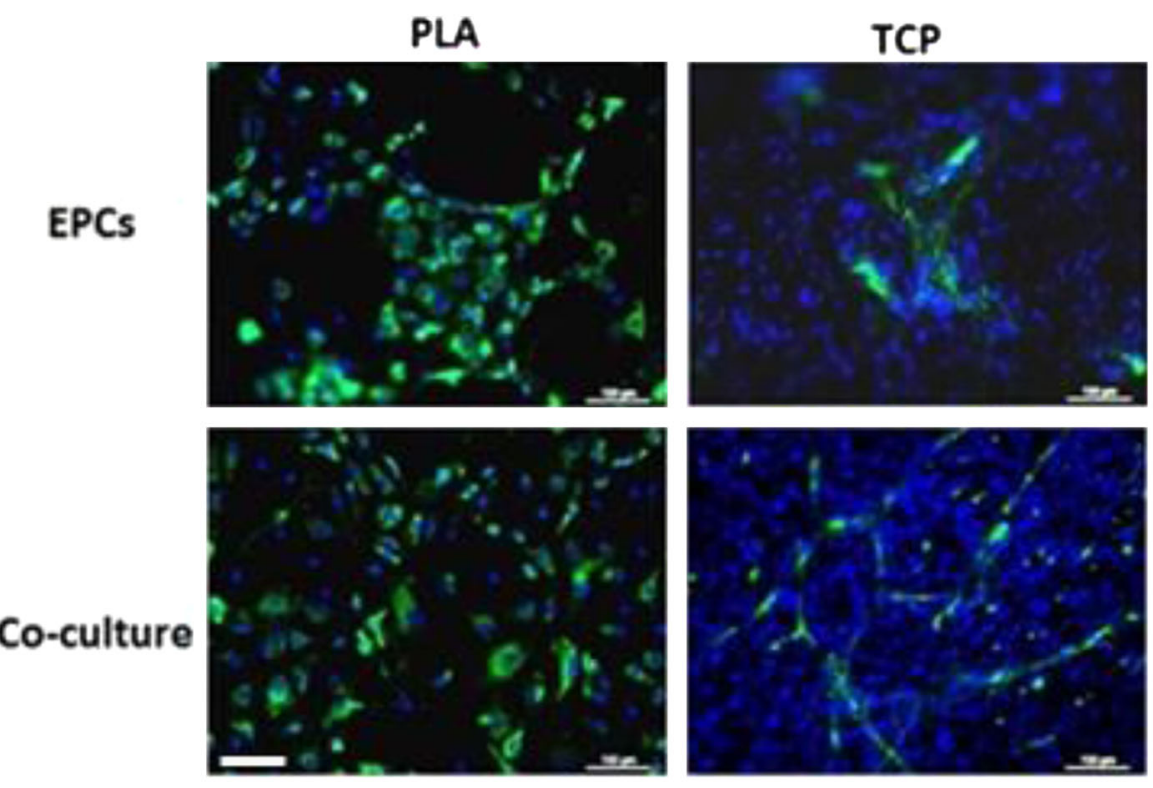

B
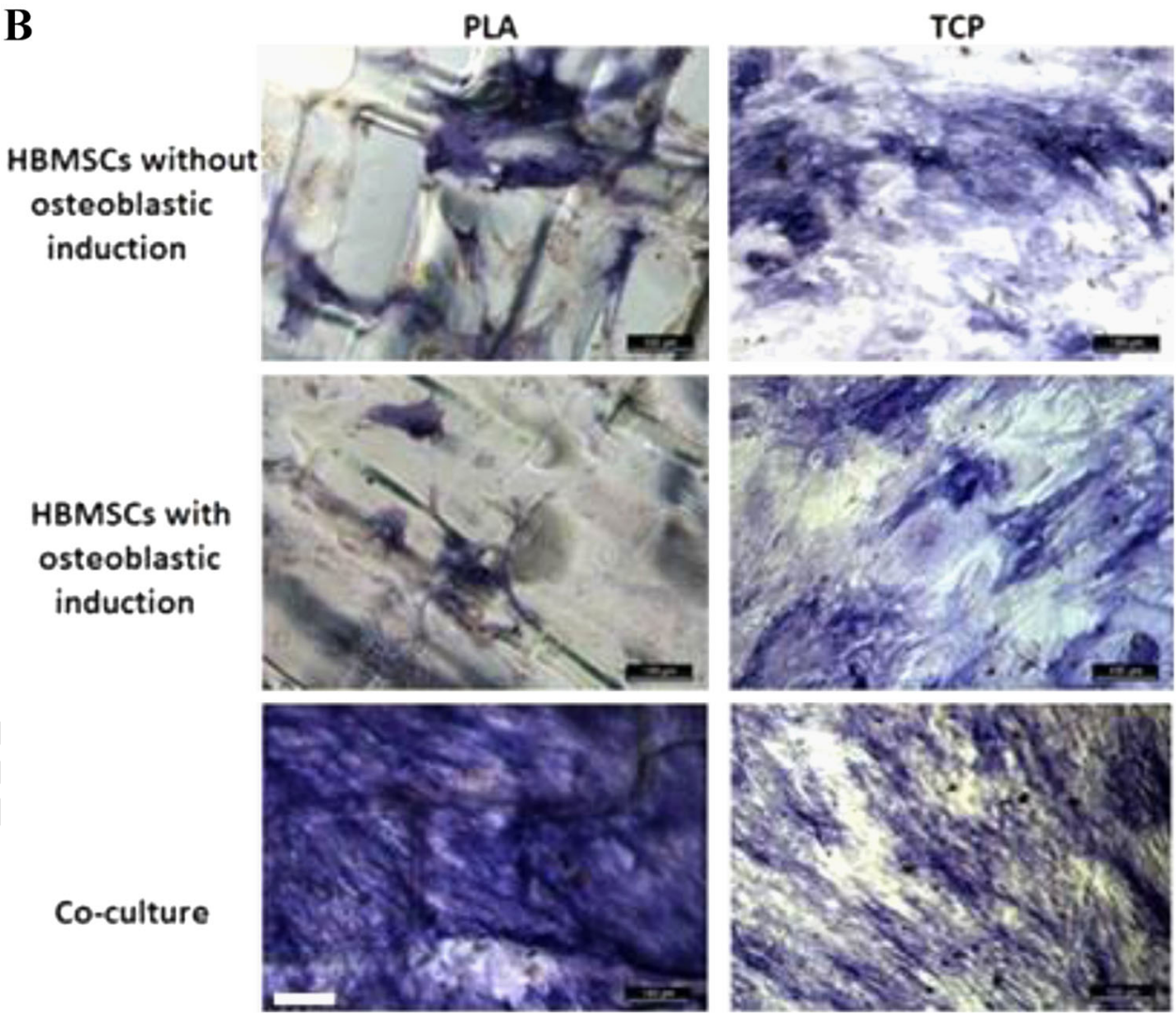

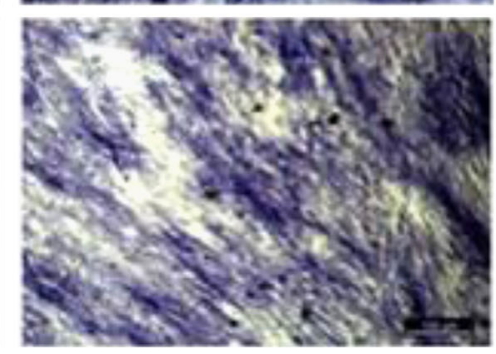

\section{Discussion}

PLA used for this work has already been characterized by Serra et al. [41]. PLA membranes fabricated by 3D printing had an expected morphology and a pore size suitable for tissue engineering [42]. Human primary cells seeded on these PLA porous membranes have shown the morphology expected in these culture conditions.
A large amount of living cells were present on PLA membranes after 14 days of culture, especially in the case of co-cultures. There were much more membrane areas covered by live than by dead cells. The highest percentage of live cells was present in co-culture systems and it increased with time, which confirmed results obtained by SEM. The presence of both types of cells provided better conditions for cell survival. There were significantly less 


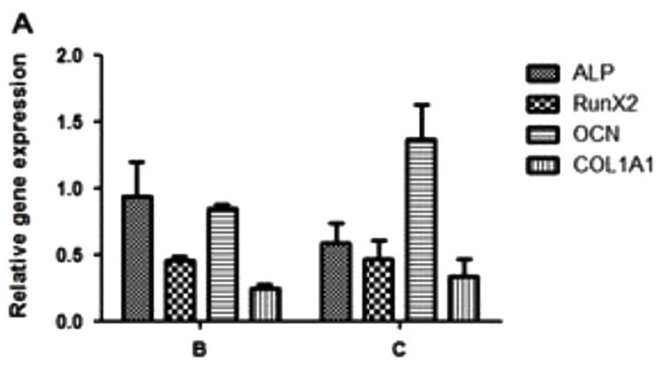

B

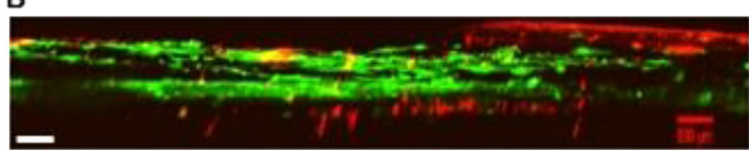

Fig. 6 3D LBL constructs. a Osteoblastic differentiation (qPCR) of cells in 3D LBL B and C types of constructs on Day 7 in comparison to the A type; b Cell colonization inside the LBL D constructs (HBMSCs-GFP in green color and EPCs-TdT in red fluorescence). The scale bar is $500 \mu \mathrm{m}$ (color figure online)

live EPCs in all conditions compared to HBMSCs and cocultures. However, the quantification of dead cells surface is not fully reliable as they usually detach from their substrate.

The amount of DNA was higher for EPCs during the first week of culture, which was expecting since we have seeded more EPCs at day 0 because they are much smaller than HBMSCs. Cells proliferation was significantly higher in the positive controls (tissue culture plastic) than on the PLA saples, what was expected with this reference tissue culture surface. There were no significant differences observed during the co-culture control samples because cell achieved their confluence very fast thanks to the cell-to-cell communication and the growth factor secretion, which was not the case on mono-culture samples. This process was slower in test co-culture samples on PLA during 7 days, but it was changed after 14 days of culture. The reason is most likely related to cell-to-cell interaction through growth factors (BMP-2, VEGF, IGF) production in co-cultures [43]. The proliferation in mono-culture samples was decreased after 7 days of culture probably because cells need more time to be adapted to the PLA than in control samples. But the proliferation was increased after 14 days, with a significant difference for HBMSCs.

EPCs were located only on struts of the PLA membranes and they formed a homogenous "grid line" shape after 14 days of culture. Co-cultures showed a higher density of cells and a lower density of vWF than mono-cultures

ALP expression was positive in both, mono- and co-cultures, which displayed early osteoblastic differentiation. The mono-cultures of HBMSCs on PLA showed similar ALP level with or without osteoblastic induction after 14 days. ALP was concentrated on the struts of the membranes. In the co-cultures performed on PLA, ALP staining covered all the surface of the membranes and pores. The ALP expression was especially high for co-cultures, which has already been described using co-cultures of HBMSCs and EPCs [44], probably because of the higher production of the extracellular matrix.

We have observed that the highest cell proliferation and viability in 2D on PLA appeared in the case of co-culture system. Then we have performed layer-by-layer bioassembly of cellularized membranes in 3D: All tridimensional LBL constructs were made of four layers of PLA membranes seeded with human primary cells. Even if we have used glass rings to stabilize the $3 \mathrm{D}$ constructs in culture plates, the materials were difficult to manipulate. Other groups have proposed to use of stainless steel mesh clips to stabilize the LBL constructs after the assembly [29]. Since we could observe the most efficient cell proliferation in coculture samples in $2 \mathrm{D}$, we decided to test osteoblastic genes expressions in culture simples with combination of 2 cell types with their different organization in aim to see if their 3D organization has an influence in osteoblastic differentiation. Control simple was mono-culture HBMSCs LBL construct (without EPCs). We have observed that OCN

and ALP had the highest relative gene expression for both LBL types. It was expected since it has already been known that they genes are expressed earlier than others. The expressions of RunX2 and Col1 were lower. But we have not observed any significant difference between the two different LBL constructs concerning the expression of osteoblastic genes. There was no difference between two different types of LBL constructs containing EPCs.

Since the positions and different combinations of HBMSCs with EPCs in layers did not play an important role in osteoblastic differentiation, we have done new LBL constructs to observe the colonization of cells inside the layers. Cells were tagged in order to observe their migration between layers of PLA. The HBMSCs were tagged by GFP (green fluorescence) and EPCs were tagged by $\mathrm{Td}$ Tomato (red fluorescence). The tested 3D construct had alternating layers of monocultures HBMSCs-GFP and co-cultures HBMSCs-GFP + EPCsTdT. Red color was present in all layers meaning that EPCs have probably migrated inside the LBL constructs.

\section{Conclusions and perspectives}

Fabrication of thin porous PLA membranes by direct 3D printing was successfully performed. Evaluations of viability, phenotypes maintain and proliferation of human primary cells cultured on PLA were positive: Cell proliferation increased with time in both, mono- and co-culture 
conditions. The level of ALP expression was higher in coculture systems. We successfully made LBL constructs by assembling four layers of cellularized PLA membranes. Experiments of these 3D constructs have shown an osteoblastic differentiation after 7 days of culture as well as the cell colonization inside the constructs. This showed the potential of LBL approach to promote a homogenous cell distribution inside the scaffold. 3D experiments have shown that LBL bio-assembly enables better cell proliferation and differentiation into the scaffold than conventional BTE. Results obtained indicate that LBL approach could be suitable for bone tissue engineering, in order to promote homogenous cell distribution into the scaffold.

Acknowledgements The authors wish to thank the French Institute in Belgrade, Serbia, via Campus France agency. 2-photon observations were done at Bordeaux Imaging Center, France.

\section{References}

1. Arealis G, Nikolaou VS. Bone printing: new frontiers in the treatment of bone defects. Injury. 2015;46(Suppl 8):S20-2.

2. O'Brien FJ. Biomaterials and scaffolds for tissue engineering,. Mater Today. 2011;14(3):88-95.

3. Oliveira $\mathrm{H}$, et al. The proangiogenic potential of a novel calcium releasing biomaterial: impact on cell recruitment. Acta Biomater. 2016;29:435-45.

4. Feng T, Liu Y, Xu Q, Li X, Luo X, Chen Y. In vitro experimental study on influences of final degradation products of polyactic acid on proliferation and osteoblastic phenotype of osteoblast-like cells. J Repar Reconstr Surg. 2014;28(12):1525-9.

5. Saito E, Suarez-Gonzalez D, Murphy WL, Hollister SJ. Biomineral coating increases bone formation by ex vivo BMP-7 gene therapy in rapid prototyped poly(L-lactic acid) (PLLA) and poly $(\varepsilon-$ caprolactone) (PCL) porous scaffolds. Adv Healthc Mater. 2015;4 (4):621-32.

6. Ciocca L, De Crescenzio F, Fantini M, Scotti R. CAD/CAM and rapid prototyped scaffold construction for bone regenerative medicine and surgical transfer of virtual planning: a pilot study. Comput Med Imaging Graph. 2009;33(1):58-62.

7. Mangano $\mathrm{F}$, et al. Maxillary ridge augmentation with custommade CAD/CAM scaffolds. A 1-year prospective study on 10 patients. J Oral Implantol. 2014;40(5):561-9.

8. Nga NK, Hoai TT, Viet PH. Biomimetic scaffolds based on hydroxyapatite nanorod/poly(D,L) lactic acid with their corresponding apatite-forming capability and biocompatibility for bone-tissue engineering. Colloids Surf B. 2015;128:506-14.

9. Lou T, Wang X, Song G, Gu Z, Yang Z. Fabrication of PLLA/ $\beta$ TCP nanocomposite scaffolds with hierarchical porosity for bone tissue engineering. Int J Biol Macromol. 2014;69:464-70.

10. D'Alessandro D, et al. Processing large-diameter poly(L-lactic acid) microfiber mesh/mesenchymal stromal cell constructs via resin embedding: an efficient histologic method. Biomed Mater Bristol Engl. 2014;9(4):045007

11. Zamparelli A, et al. Growth on poly(L-lactic acid) porous scaffold preserves CD73 and CD90 immunophenotype markers of rat bone marrow mesenchymal stromal cells. J Mater Sci Mater Med. 2014;25(10):2421-36.

12. Kao C-T, Lin C-C, Chen Y-W, Yeh C-H, Fang H-Y, Shie M-Y. Poly(dopamine) coating of 3D printed poly(lactic acid) scaffolds for bone tissue engineering. Mater Sci Eng C. 2015; $56: 165-73$

13. Hu Y, Zou S, Chen W, Tong Z, Wang C. Mineralization and drug release of hydroxyapatite/poly(L-lactic acid) nanocomposite scaffolds prepared by pickering emulsion templating. Colloids Surf B Biointerfaces. 2014;122:559-65.

14. Ding M, Henriksen SS, Wendt D, Overgaard S. An automated perfusion bioreactor for the streamlined production of engineered osteogenic grafts. J Biomed Mater Res B. 2015;104:532-537.

15. Lian Q, Zhuang P, Li C, Jin Z, Li D. Mechanical properties of polylactic acid/beta-tricalcium phosphate composite scaffold with double channels based on three-dimensional printing technique. Chin J Repar Reconstr Surg. 2014;28(3):309-13.

16. Ronca A, et al. Large defect-tailored composite scaffolds for in vivo bone regeneration. J Biomater Appl. 2014;29(5):715-27.

17. Hamad K. Properties and medical applications of polylactic acid: a review. Express Polym Lett. 2015;9(5):435-55.

18. Vidyasekar P, Shyamsunder P, Sahoo SK, Verma RS. Scaffoldfree and scaffold-assisted 3D culture enhances differentiation of bone marrow stromal cells. In Vitro Cell Dev Biol Anim. 2016;52 (2):204-17.

19. Huang J, et al. Evaluation of the novel three-dimensional porous poly (L-lactic acid)/nano-hydroxyapatite composite scaffold. Biomed Mater Eng. 2015;26(Suppl 1):S197-205.

20. Giordano RA, Wu BM, Borland SW, Cima LG, Sachs EM, Cima MJ. Mechanical properties of dense polylactic acid structures fabricated by three dimensional printing. J Biomater Sci Polym Ed. 1996;8(1):63-75.

21. Almeida CR, Serra T, Oliveira MI, Planell JA, Barbosa MA, Navarro M. Impact of 3-D printed PLA- and chitosan-based scaffolds on human monocyte/macrophage responses: unraveling the effect of 3-D structures on inflammation. Acta Biomater. 2014;10(2):613-22.

22. Serra T, Mateos-Timoneda MA, Planell JA, Navarro M. 3D printed PLA-based scaffolds: a versatile tool in regenerative medicine. Organogenesis. 2013;9(4):239-44.

23. Schlaubitz S, et al. Pullulan/dextran/nHA macroporous composite beads for bone repair in a femoral condyle defect in rats. PLoS One. 2014;9(10):e110251

24. Groll J, et al. Biofabrication: reappraising the definition of an evolving field. Biofabrication. 2016;8(1):013001

25. Sathy BN, Mony U, Menon D, Baskaran VK, Mikos AG, Nair S. Bone tissue engineering with multilayered scaffolds-part $\mathrm{I}$ : an approach for vascularizing engineered constructs in vivo. Tissue Eng Part A. 2015;21(19-20):2480-94.

26. Ren L, et al. Preparation of three-dimensional vascularized MSC cell sheet constructs for tissue regeneration. BioMed Res Int. 2014;2014:301279

27. Nishiguchi A, Matsusaki M, Asano Y, Shimoda H, Akashi M. Effects of angiogenic factors and 3D-microenvironments on vascularization within sandwich cultures. Biomaterials. 2014;35 (17):4739-48.

28. Derda R, et al. Paper-supported 3D cell culture for tissue-based bioassays. Proc Natl Acad Sci USA. 2009;106(44):18457-62.

29. Wan W, et al. Layer-by-layer paper-stacking nanofibrous membranes to deliver adipose-derived stem cells for bone regeneration. Int J Nanomedicine. 2015;10:1273-90.

30. Catros $S$, et al. Layer-by-layer tissue microfabrication supports cell proliferation in vitro and in vivo. Tissue Eng Part C Methods. 2012;18(1):62-70.

31. Wen $\mathrm{L}$, et al. Role of endothelial progenitor cells in maintaining stemness and enhancing differentiation of mesenchymal stem cells by indirect cell-cell interaction. Stem Cells Dev. 2016;25(2):123-38.

32. Eldesoqi K, et al. Safety evaluation of a bioglass-polylactic acid composite scaffold seeded with progenitor cells in a rat skull critical-size bone defect. PLoS One. 2014;9(2):e87642 
33. Vilamitjana-Amedee J, Bareille R, Rouais F, Caplan AI, Harmand MF. Human bone marrow stromal cells express an osteoblastic phenotype in culture. In Vitro Cell Dev Biol Anim. 1993;29A (9):699-707.

34. Thebaud NB, Bareille R, Remy M, Bourget C, Daculsi R, Bordenave L. Human progenitor-derived endothelial cells vs. venous endothelial cells for vascular tissue engineering: an in vitro study. J Tissue Eng Regen Med. 2010;4(6):473-84.

35. Thébaud NB, et al. Labeling and qualification of endothelial progenitor cells for tracking in tissue engineering: an in vitro study. Int J Artif Organs. 2015;38(4):224-32.

36. Lau KR, Evans RL, Case RM. Intracellular Cl- concentration in striated intralobular ducts from rabbit mandibular salivary glands. Pflüg Arch Eur J Physiol. 1994;427(1-2):24-32.

37. Poole CA, Brookes NH, Clover GM. Keratocyte networks visualised in the living cornea using vital dyes. J Cell Sci. 1993;106(Pt 2):685-91.

38. Vaughan PJ, Pike CJ, Cotman CW, Cunningham DD. Thrombin receptor activation protects neurons and astrocytes from cell death produced by environmental insults. J Neurosci. 1995;15 (7):5389-401. Pt 2
39. Metcalf DJ, Nightingale TD, Zenner HL, Lui-Roberts WW, Cutler DF. Formation and function of Weibel-Palade bodies. J Cell Sci. 2008;121(Pt 1):19-27.

40. Szczurek AT, et al. Single molecule localization microscopy of the distribution of chromatin using Hoechst and DAPI fluorescent probes. Nucl Austin Tex. 2014;5(4):331-40.

41. Serra T, Ortiz-Hernandez M, Engel E, Planell JA, Navarro M. Relevance of PEG in PLA-based blends for tissue engineering 3Dprinted scaffolds. Mater Sci Eng C. 2014;38:55-62.

42. Ahn S, Lee H, Kim G. Functional cell-laden alginate scaffolds consisting of core/shell struts for tissue regeneration. Carbohydr Polym. 2013;98(1):936-42.

43. Aguirre A, Planell JA, Engel E. Dynamics of bone marrowderived endothelial progenitor cell/mesenchymal stem cell interaction in co-culture and its implications in angiogenesis. Biochem Biophys Res Commun. 2010;400(2):284-91.

44. Grellier M, Bordenave L, Amédée J. Cell-to-cell communication between osteogenic and endothelial lineages: implications for tissue engineering. Trends Biotechnol. 2009;27(10):562-71. 


\section{Springer}

the language of science

\section{Author Query Form}

\section{Please ensure you fill out your response to the queries raised below and return this form along with your corrections}

\section{Dear Author}

During the process of typesetting your article, the following queries have arisen. Please check your typeset proof carefully against the queries listed below and mark the necessary changes either directly on the proof/online grid or in the 'Author's response' area provided below

\begin{tabular}{|c|l|l|}
\hline Queries & Details Required & Author's Response \\
\hline AQ1 & Please check and confirm the given corresponding author is ok. & \\
\hline AQ2 & Please provide 'conflict of interest' statement. & \\
\hline
\end{tabular}

